\title{
Cerebral Arterial Stenosis in Patients with Spontaneous Intracerebral Hemorrhage
}

\author{
Pil-Wook Chung, M.D., Ph.D., ${ }^{1}$ Yu Sam Won, M.D., Ph.D. ${ }^{2}$ \\ Departments of Neurology, Neurosurgery, Kangbuk Samsung Hospital, Sungkyunkwan University School of Medicine, Seoul, Korea
}

Objective : Spontaneous intracerebral hemorrhage $(\mathrm{ICH})$ and ischemic stroke share common vascular risk factors such as aging and hypertension. Previous studies suggested that the rate of recurrent $\mathrm{ICH}$ and ischemic stroke might be similar after ICH. Presence of cerebral arterial stenosis is a potential risk factor for future ischemic stroke. This study investigated the prevalence and factors associated with cerebral arterial stenosis in Korean patients with spontaneous $\mathrm{ICH}$.

Methods : A total of 167 patients with spontaneous ICH were enrolled. Intracranial arterial stenosis (ICAS) and extracranial arterial stenosis (ECAS) were assessed by computed tomography angiography. Presence of ICAS was defined if patients had arterial stenosis in at least one intracranial artery. ECAS was assessed in the extracranial carotid artery. More than $50 \%$ luminal stenosis was defined as presence of stenosis. Prevalence and factors associated with presence of ICAS and cerebral arterial stenosis (presence of ICAS and/or ECAS) were investigated by multivariable logistic regression analysis.

Results : Thirty-two (19.2\%) patients had ICAS, 7.2\% had ECAS, and 39 (23.4\%) patients had any cerebral arterial stenosis. Frequency of ICAS and ECAS did not differ among ganglionic ICH, lobar ICH, and brainstem ICH. Age was higher in patients with ICAS ( $67.6 \pm 11.8$ vs. $58.9 \pm 13.6$ years $p=0.004)$ and cerebral arterial stenosis $(67.9 \pm 11.6$ vs. $59.3 \pm 13.5$ years, $p<0.001)$ compared to those without stenosis. Patients with ICAS were older, more frequently had diabetes, had a higher serum glucose level, and had a lower hemoglobin level than those without ICAS. Patients with cerebral arterial stenosis were older, had diabetes and lower hemoglobin level, which was consistent with findings in patients with ICAS. However, patients with cerebral arterial stenosis showed higher prevalence of hypertension and decreased kidney function compared to those without cerebral arterial stenosis. Multivariable logistic regression analyses showed that aging and presence of diabetes independently predicted the presence of ICAS, and aging, diabetes, and hypertension were independently associated with presence of cerebral arterial stenosis.

Conclusion : $19.2 \%$ of patients with spontaneous ICH had ICAS, but the prevalence of ECAS was relatively lower (7.2\%) compared with ICAS. Aging and diabetes were independent factors for the presence of ICAS, whereas aging, hypertension, and diabetes were factors for the cerebral arterial stenosis.

Key Words : Cerebral hemorrhage · Diabetes · Hypertension · Cerebral arterial stenosis · Computed tomography angiography.

- Received : November 1, 2016 •Revised : February 6, 2017 •Accepted : April 11, 2017

- Address for reprints : Yu Sam Won, M.D., Ph.D.

Department of Neurosurgery, Kangbuk Samsung Hospital, Sungkyunkwan University School of Medicine, 29 Saemunan-ro, Jongno-gu, Seoul 03181, Korea Tel : +82-2-2001-2159, Fax : +82-2-2001-2157, E-mail : yusam.won@samsung.com

This is an Open Access article distributed under the terms of the Creative Commons Attribution Non-Commercial License (http://creativecommons.org/licenses/by-nc/4.0) which permits unrestricted non-commercial use, distribution, and reproduction in any medium, provided the original work is properly cited. 


\section{INTRODUCTION}

Spontaneous intracerebral hemorrhage (ICH) usually results from the rupture of small arteries in the brain and represents $10-30 \%$ of all strokes ${ }^{20)}$. ICH is associated with high rate of mortality and long-term disability in survivors. ICH and ischemic stroke share common vascular risk factors such as aging and hypertension. Secondary prevention of recurrence is the main treatment goal after prior stroke for both ischemic and hemorrhagic stroke. Importantly, several studies reported no differences of frequency of hemorrhagic or ischemic stroke recurrence after prior $\mathrm{ICH}^{7,8,26)}$. However, risk factors and features of recurrent stroke after ICH are not firmly established. Extracranial carotid stenosis is a well-known risk factor for stroke. Furthermore, it is widely accepted that intracranial cerebral arterial stenosis (ICAS) is more prevalent in Asians than in Caucasian stroke population, and $30-50 \%$ of stroke in Asians is suggested to be caused by ICAS ${ }^{3,25)}$. Therefore, detection of cerebral arterial stenosis, such as ICAS and extracranial cerebral arterial stenosis (ECAS) may have clinical significance for secondary stroke prevention in ICH patients. Although a previous study suggested that ICAS or ECAS occur in one-fifth of patients with $\mathrm{ICH}^{22}$, little is known of the prevalence and factors associated with cerebral arterial stenosis in patients with ICH.

We investigated the prevalence of ICAS and ECAS, and analyzed the factors predicting the presence of arterial stenosis in patients presenting with spontaneous ICH.

\section{MATERIALS AND METHODS}

\section{Patients}

Patients with spontaneous ICH who were admitted to a tertiary university hospital were included. Patients were retrospectively identified from the neurosurgical database for ICH between January 2009 to December 2012. The inclusion criteria were as follows : 1) spontaneous ICH within 24 hours of presumed symptom onset, 2) patients underwent computed tomography angiography (CTA), and 3) age $>18$ years. The exclusion criteria were ICH due to secondary causes like arteriovenous malformation, Moyamoya disease, traumatic $\mathrm{ICH}$, aneurysm, neoplasm, and hemorrhagic conversion of cerebral infarction. The local institutional review board ap- proved this retrospective study and waived the need for informed consent.

\section{CTA and cerebral arterial stenosis}

CTA was used to analyze the presence of intracranial and extracranial stenosis. All patients underwent multi-detector CTA, which was performed using a Brilliance 40-channel CT v2.2 (Philips Medical System, Best, The Netherlands) with the following parameters : $120 \mathrm{kVp}, 300 \mathrm{~mA}, 0.8 \mathrm{~mm}$ slice thickness, $0.3 \mathrm{~mm}$ slice acquisition interval, a pitch of 0.67 , and intravenous administration of $150 \mathrm{~mL}$ of iodinated contrast media (Omnipasque 300) at a rate of 4.0-4.5 mL/s under the control of an auto-injection protocol. Imaging data were transferred to two computer workstations (Extended Brilliance Workspace v3.0 and Rapidia v2.8) for post-processing.

Stenosis was determined as the ratio of the diameter at the segment of most severe stenosis divided by the diameter of a proximal or nearby normal segment ${ }^{21)}$. More than $50 \%$ stenosis was defined as arterial stenosis. We analyzed stenosis for intracranial artery and extracranial artery. Intracranial arteries that were evaluated were the intracranial segment of internal carotid artery, basilar artery, anterior cerebral artery, middle cerebral artery, and posterior cerebral artery. Extracranial stenosis was analyzed in the extracranial portion of the internal carotid artery. We excluded the extracranial and intracranial vertebral arteries from this analysis since the hypoplastic vertebral arteries are frequently found in normal subjects, which make it difficult to differentiate between non-specific hypoplasia and atherosclerotic stenosis. Location of ICH were categorized as lobar (cortical and subcortical), ganglionic (putamen and thalamus), and infratentorial (brainstem and cerebellum).

\section{Clinical characteristics}

The Glasgow coma scale was recorded to assess the initial neurological status on admission, and patient's functional outcome at discharge and 90 days were assessed using the Glasgow outcome scale (1 to 5 , good recovery to death). Hypertension was defined as a self-reported use of blood pressure-lowering medication or history of hypertension or blood pressure $>160 / 100 \mathrm{mmHg}$ at least 1 week after stroke onset. Diabetes mellitus was defined present if patient was taking hypoglycemic medication or had a random glucose 
level $\geq 200 \mathrm{mg} / \mathrm{dL}$. Dyslipidemia was defined when using anti-lipidemic agent or total cholesterol level $\geq 220 \mathrm{mg} / \mathrm{dL}$ or low-density lipoprotein cholesterol level $\geq 160 \mathrm{mg} / \mathrm{dL}$. Current cigarette smoking was considered a positive smoking habitus. The estimated glomerular filtration rate (eGFR) was calculated using the equation proposed by the MDRD study group ${ }^{13)}$.

\section{Statistical analysis}

Baseline characteristics and location of ICH were compared between patients with ICAS and without ICAS. We also compared patients with cerebral arterial stenosis (defined as presence of ICAS and/or ECAS) and those without cerebral arterial stenosis. Baseline characteristics and location of $\mathrm{ICH}$ were compared using chi-square test and the Student t-test, as appropriate. Multivariable analyses were performed via multiple logistic regression to determine the factors associated with presence of ICAS and cerebral arterial stenosis. In multivariable analysis, variables with $p<0.2$ from the univariate analysis

Table 1. Baseline characteristics

\begin{tabular}{|c|c|}
\hline & Value $(n=167)$ \\
\hline Female sex & $65(38.9)$ \\
\hline Age (years) & $61.3 \pm 13.6$ \\
\hline Hypertension & $99(59.3)$ \\
\hline Diabetes & $28(16.8)$ \\
\hline Dyslipidemia & $32(19.2)$ \\
\hline Previous stroke & $29(17.4)$ \\
\hline Current smoking & $39(23.4)$ \\
\hline Initial GCS & $12.6 \pm 3.3$ \\
\hline Discharge GOS & $3.3 \pm 0.7$ \\
\hline 3 months GOS & $3.4 \pm 0.9$ \\
\hline Leukocyte $\left(\mathrm{mm}^{3}\right)$ & $9244 \pm 3874$ \\
\hline Hemoglobin (g/dL) & $14.1 \pm 1.8$ \\
\hline Serum glucose (mg/dL) & $148.1 \pm 53.1$ \\
\hline Total cholesterol (mg/dL) & $182.5 \pm 44.4$ \\
\hline HDL cholesterol (mg/dL) & $55.0 \pm 18.6$ \\
\hline LDL cholesterol (mg/dL) & $111.5 \pm 40.7$ \\
\hline Triglyceride (mg/dL) & $105.8 \pm 81.2$ \\
\hline Serum creatinine (mg/dL) & $0.93 \pm 0.48$ \\
\hline $\mathrm{eGFR}\left(\mathrm{mL} / \mathrm{min} / 1.73 \mathrm{~m}^{2}\right)$ & $89.7 \pm 28.5$ \\
\hline
\end{tabular}

Values are presented as mean \pm standard deviation or number (\%). GCS : Glasgow coma scale, GOS : Glasgow outcome scale, HDL : high-density lipoprotein, LDL : low-density lipoprotein, eGFR : estimated glomerular filtration rate and biologically plausible variables were selected as potential factors for statistical analysis. All analyses were performed using PASW statistics version 18.0 (IBM Corp., Armonk, NY, USA).

\section{RESULTS}

Present study included 187 patients with spontaneous ICH. Of them, 20 patients who did not undergo CTA were excluded from this analysis. The main reason for not performing CTA was irritability and/or unstable patient condition due to acute stroke. Therefore, 167 patients were finally included. Mean age was $61.3 \pm 13.6$ years. Sixty five (38.9\%) were female, $59.3 \%$ had hypertension, $16.8 \%$ had diabetes, $19.2 \%$ had dyslipidemia, and $23.4 \%$ were current smoker (Table 1).

Among the 167 patients who underwent CTA, 32 (19.2\%) had at least one ICAS, and 15 (9\%) had multiple ICAS (Table 2). Extracranial carotid stenosis was detected in 12 (7.2\%) patients, and 39 (23.4\%) patients had cerebral arterial stenosis (ICAS and/or ECAS). Patients with ICAS were older, more frequently had diabetes, and had a higher initial serum glucose level than those without ICAS. Hemoglobin level was lower in patients with ICAS (Table 3). However, other vascular risk factors and laboratory finding did not differ between the two groups. Location of hemorrhage (lobar, ganglionic, and infratentorial) and volume of ICH also did not differ between the patients with and without ICAS. Patients with cerebral arterial stenosis (ICAS and/or ECAS) were older, had diabetes

Table 2. Radiological features

\begin{tabular}{lc}
\hline & Value $(\mathbf{n = 1 6 7 )}$ \\
\hline Location of ICH & \\
Ganglionic & $118(70.6)$ \\
Lobar & $28(16.8)$ \\
Brainstem/cerebellum & $21(12.6)$ \\
Intracranial stenosis & $32(19.2)$ \\
Middle cerebral artery & $19(11.4)$ \\
Posterior cerebral artery & $2(1.2)$ \\
Internal carotid artery & $12(7.2)$ \\
Basilar artery & $6(3.6)$ \\
Multiple stenosis & $15(9)$ \\
Extracranial stenosis & $12(7.2)$ \\
\hline
\end{tabular}

Values are presented as number (\%). ICH : intracerebral hemorrhage 
and lower hemoglobin level, which is consistent with the findings of patients with ICAS. However, patients with cerebral arterial stenosis had higher prevalence of hypertension and decreased kidney function estimated by eGFR compared to those without cerebral arterial stenosis (Table 4).

Multivariable logistic regression analyses showed that aging and presence of diabetes independently predicted the presence of ICAS. However, other factors including hypertension and dyslipidemia were not associated with the presence of ICAS (Table 5). Factors independently predicting the presence of cerebral arterial stenosis using multivariable logistic regression

Table 3. Comparison between patients with intracranial stenosis and those without stenosis

\begin{tabular}{|c|c|c|c|}
\hline & $\begin{array}{l}\text { With stenosis } \\
\qquad(\mathrm{n}=32)\end{array}$ & $\begin{array}{l}\text { Without } \\
\text { stenosis } \\
(n=135)\end{array}$ & $p$-value \\
\hline Age (years) & $67.6 \pm 11.8$ & $58.9 \pm 13.6$ & 0.004 \\
\hline Female sex & $14(43.8)$ & $51(37.8)$ & 0.533 \\
\hline Hypertension & $24(75)$ & $75(56.8)$ & 0.059 \\
\hline Diabetes & $11(34.4)$ & $17(12.9)$ & 0.004 \\
\hline Dyslipidemia & $7(21.9)$ & $25(18.9)$ & 0.707 \\
\hline Previous stroke & $6(18.8)$ & $23(17.4)$ & 0.86 \\
\hline Current smoking & $6(18.8)$ & $33(25)$ & 0.456 \\
\hline Initial GCS & $12.4 \pm 3.6$ & $12.6 \pm 3.2$ & 0.83 \\
\hline Discharge GOS & $3.1 \pm 0.9$ & $3.4 \pm 0.7$ & 0.062 \\
\hline 3 months GOS & $3.1 \pm 1.1$ & $3.5 \pm 0.8$ & 0.014 \\
\hline Initial SBP (mmHg) & $183 \pm 35$ & $174 \pm 38$ & 0.273 \\
\hline Leukocyte $\left(\mathrm{mm}^{3}\right)$ & $8887 \pm 5248$ & $9329 \pm 3486$ & 0.653 \\
\hline Hemoglobin (g/dL) & $13.5 \pm 1.9$ & $14.2 \pm 1.8$ & 0.033 \\
\hline Serum glucose (mg/dL) & $168 \pm 52$ & $143 \pm 52$ & 0.017 \\
\hline Total cholesterol (mg/dL) & $177 \pm 44$ & $183 \pm 45$ & 0.459 \\
\hline HDL cholesterol (mg/dL) & $52 \pm 13.6$ & $55.7 \pm 19.6$ & 0.327 \\
\hline LDL cholesterol (mg/dL) & $110.8 \pm 39.5$ & $111.7 \pm 41.1$ & 0.912 \\
\hline Triglyceride (mg/dL) & $92.5 \pm 76.8$ & $108.9 \pm 82.2$ & 0.327 \\
\hline Serum creatinine (mg/dL) & $1.2 \pm 0.9$ & $0.9 \pm .02$ & 0.102 \\
\hline $\operatorname{eGFR}\left(\mathrm{mL} / \mathrm{min} / 1.73 \mathrm{~m}^{2}\right)$ & $81.8 \pm 36.7$ & $91.5 \pm 25.9$ & 0.082 \\
\hline Location of ICH & & & 0.981 \\
\hline Ganglionic & 23 & 95 & \\
\hline Lobar & 5 & 23 & \\
\hline Brainstem/Cerebellum & 4 & 17 & \\
\hline
\end{tabular}

Values are presented as mean \pm standard deviation or number (\%). GCS : Glasgow coma scale, GOS : Glasgow outcome scale, SBP : systolic blood pressure, HDL : high-density lipoprotein, LDL : low-density lipoprotein, eGFR : estimated glomerular filtration rate analysis were aging, diabetes, and hypertension Multivariable logistic regression analysis for ECAS was not done since the sample size of patients with ECAS ( $\mathrm{n}=12$ ) was not enough to demonstrate the effect in multivariable modeling.

\section{DISCUSSION}

Present study shows that $19.2 \%$ of patients with spontaneous ICH had ICAS. Multivariable analysis identified aging

Table 4. Comparison between patients with cerebral arterial stenosis (presence of intracranial and/or extracranial stenosis) and those without cerebral arterial stenosis

\begin{tabular}{|c|c|c|c|}
\hline & $\begin{array}{l}\text { With stenosis } \\
\qquad(n=39)\end{array}$ & $\begin{array}{l}\text { Without } \\
\text { stenosis } \\
(n=128)\end{array}$ & $p$-value \\
\hline Age (years) & $67.9 \pm 11.6$ & $59.3 \pm 13.5$ & $<0.001$ \\
\hline Female sex & $15(38.5)$ & $50(39.1)$ & 0.946 \\
\hline Hypertension & $29(74.4)$ & $70(54.7)$ & 0.041 \\
\hline Diabetes & $13(33.3)$ & $15(12)$ & 0.002 \\
\hline Dyslipidemia & $9(23.1)$ & $23(18)$ & 0.52 \\
\hline Previous stroke & $8(20.5)$ & $21(16.4)$ & 0.596 \\
\hline Smoking current & $9(23.1)$ & $30(23.4)$ & 0.906 \\
\hline Initial GCS & $12.5 \pm 3.4$ & $12.6 \pm 3.2$ & 0.982 \\
\hline Discharge GOS & $3.2 \pm 0.8$ & $3.4 \pm 0.7$ & 0.13 \\
\hline 3month GOS & $3.2 \pm 0.9$ & $3.5 \pm 0.8$ & 0.039 \\
\hline Initial SBP (mmHg) & $182 \pm 36$ & $174 \pm 38$ & 0.26 \\
\hline Leukocyte $\left(\mathrm{mm}^{3}\right)$ & $8980 \pm 5077$ & $9326 \pm 3442$ & 0.692 \\
\hline Hemoglobin (g/dL) & $13.6 \pm 1.8$ & $14.2 \pm 1.8$ & 0.046 \\
\hline Serum glucose (mg/dL) & $164 \pm 54$ & $143 \pm 52$ & 0.034 \\
\hline Total cholesterol (mg/dL) & $181 \pm 48$ & $183 \pm 43$ & 0.794 \\
\hline HDL cholesterol (mg/dL) & $54.8 \pm 15.6$ & $55.0 \pm 19.4$ & 0.946 \\
\hline LDL cholesterol (mg/dL) & $112.2 \pm 45.2$ & $111.3 \pm 39.4$ & 0.913 \\
\hline Triglyceride (mg/dL) & $90.7 \pm 75.1$ & $110.4 \pm 82.7$ & 0.21 \\
\hline Serum creatinine (mg/dL) & $1.1 \pm 0.9$ & $0.9 \pm 0.2$ & 0.057 \\
\hline eGFR $\left(\mathrm{mL} / \mathrm{min} / 1.73 \mathrm{~m}^{2}\right)$ & $80.2 \pm 33.7$ & $92.6 \pm 26.1$ & 0.018 \\
\hline Location of ICH & & & 0.511 \\
\hline Ganglionic & 26 & 92 & \\
\hline Lobar & 6 & 22 & \\
\hline Brainstem/Cerebellum & 7 & 14 & \\
\hline
\end{tabular}

Values are presented as mean \pm standard deviation or number (\%), GCS : Glasgow coma scale, GOS : Glasgow outcome scale, SBP : systolic blood pressure, HDL : high-density lipoprotein, LDL : low-density lipoprotein, eGFR : estimated glomerular filtration rate, ICH : intracerebral hemorrhage 
Table 5. Multivariable logistic regression analysis for presence of intracranial stenosis and cerebral arterial stenosis (presence of intracranial and/or extracranial stenosis)

\begin{tabular}{|c|c|c|c|}
\hline & OR & $95 \% \mathrm{Cl}$ & $p$-value \\
\hline \multicolumn{4}{|l|}{ Intracranial stenosis } \\
\hline Age (per 1 year) & 1.05 & $1.01-1.09$ & 0.015 \\
\hline Female sex & 0.79 & $0.29-2.17$ & 0.647 \\
\hline Hypertension & 2.49 & $0.88-7.01$ & 0.085 \\
\hline Diabetes & 3.09 & $1.12-8.56$ & 0.03 \\
\hline Dyslipidemia & 1.32 & $0.46-3.81$ & 0.607 \\
\hline Prior stroke & 0.53 & $0.16-1.71$ & 0.285 \\
\hline Smoking & 1.66 & $0.51-5.42$ & 0.4 \\
\hline Hemoglobin & 0.89 & $0.68-1.16$ & 0.38 \\
\hline eGFR & 0.99 & $0.98-1.01$ & 0.689 \\
\hline \multicolumn{4}{|c|}{ Cerebral arterial stenosis } \\
\hline Age (per 1 year) & 1.07 & $1.03-1.12$ & 0.001 \\
\hline Female sex & 0.53 & $0.20-1.41$ & 0.201 \\
\hline Hypertension & 2.95 & $1.09-7.95$ & 0.032 \\
\hline Diabetes & 3.33 & $1.21-9.16$ & 0.02 \\
\hline Dyslipidemia & 1.66 & $0.61-4.56$ & 0.323 \\
\hline Prior stroke & 0.57 & $0.18-1.74$ & 0.322 \\
\hline Smoking & 2.53 & $0.84--7.62$ & 0.099 \\
\hline Hemoglobin & 0.9 & $0.69-1.18$ & 0.445 \\
\hline eGFR & 0.99 & $0.98-1.01$ & 0.345 \\
\hline
\end{tabular}

OR : odds ratio, $\mathrm{Cl}$ : confidence interval, eGFR : estimated glomerular filtration rate

and diabetes as independent factors associated with the presence of ICAS. Compared with ICAS, extracranial carotid stenosis was less frequently found (7.2\%). Any presence of cerebral arterial stenosis (presence of ICAS and/or ECAS) was observed in $23.4 \%$ of the patients. Aging, hypertension, and diabetes were factors for the presence of cerebral atherosclerosis.

Evidence of cerebral atherosclerosis in patients with spontaneous ICH has been sparsely investigated until recently. A Japanese study reported that $13 \%$ of patients had ICAS and 9\% had ECAS (combining extracranial carotid artery and extracranial vertebral artery $)^{22}$. However, the prevalence of cerebral atherosclerosis in patients with ICH has not been reported in the western population. It is well known that ICAS is more prevalent in Asians than in Caucasian stroke population ${ }^{3)}$, and reportedly causes up to $30-50 \%$ of ischemic strokes in Asians ${ }^{25}$. Several studies investigated the prevalence of asymp- tomatic ICAS in patients presenting with ischemic stroke in another vascular territory. Coexisting asymptomatic ICAS was highly prevalent (27\%) in patients with ischemic stroke enrolled in the WASID trial ${ }^{18)}$. ICAS was reported in $10 \%$ and was symptomatic in $7 \%$ of Norwegian ischemic stroke/TIA population ${ }^{15}$. However, the prevalence of asymptomatic ICAS in subjects without stroke is not well established. Transcranial Doppler study in subjects with vascular risk factors showed that $12 \%$ had ICAS with increasing prevalence that correlated with the increasing number of vascular risk factors ${ }^{24)}$. A prevalence study of ICAS in a non-stroke population showed that aging was associated with ICAS, reflecting the strong relationship between aging and atherosclerotic burden. Although we do not know the real prevalence of ICAS in different population groups (ischemic stroke, ICH, and non-stroke), the prevalence of ICAS in patients with ICH as shown in our study is comparable to previously-reported prevalence of asymptomatic ICAS in those with ischemic stroke.

The risk of recurrent stroke in patients with symptomatic ICAS is among the highest of the stroke subtype ${ }^{11)}$. A recent randomized controlled trial in patients with ICAS reported a risk of any recurrent stroke or death of $17.5 \%$ at 1 year and $19.8 \%$ at 2 years despite best medical management ${ }^{6}$. However, despite the high risk of subsequent vascular events in patients with symptomatic ICAS, the significance of asymptomatic ICAS remains unknown. The risk of stroke from asymptomatic stenosis is thought to be relatively low ${ }^{18)}$, however, other study showed asymptomatic ICAS is significant risk factor for future stroke ${ }^{16}$. Furthermore, presence of asymptomatic ICAS may portend advanced atherosclerosis and could be a risk marker for subsequent vascular event as suggested by high prevalence of coronary artery disease in patients with symptomatic ICAS ${ }^{1,17}$. It is, therefore, possible that the presence of asymptomatic ICAS in patients with ICH may suggest higher risk of ischemic vascular event despite incident hemorrhagic stroke.

ICH and ischemic stroke share common vascular risk factors such as aging and hypertension. Recurrent stroke after prior ICH could present as either hemorrhagic or ischemic subtypes, although the epidemiology and features of recurrent stroke after ICH are unclear. Recurrent ICH has been reported to occur more frequently than ischemic stroke after prior $\mathrm{ICH}^{2,9)}$. However, other studies claimed that there are no differences of recurrence between hemorrhagic and ischemic 
stroke or numerically higher rate of recurrent ischemic stroke after prior $\mathrm{ICH}^{7,8,26)}$. Which clinical characteristics represent higher risk for ischemic stroke among ICH population is still unknown. Therefore, secondary stroke prevention strategy for reducing ischemic stroke event in patients with prior hemorrhagic stroke has not been established. Our study showed that ICH patients with older age and diabetes have higher risk for ICAS, and those with diabetes and hypertension are at higher risk for cerebral atherosclerosis, which might have a clinical implication for preventing subsequent ischemic event in patients with prior ICH.

Lobar and non-lobar ICH are presumably caused by different types of microangiopathy, and risk factors for ICH may vary according to the location of $\mathrm{ICH}^{12)}$. Regarding the location of ICH, lobar ICH frequently recurs as lobar ICH suggesting the participation of cerebral amyloid angiopathy as a main pathogenesis, but ganglionic ICH may have a chance of recurrence both as infarction and ganglionic $\mathrm{ICH}$, suggesting the participation of intracranial atherosclerosis ${ }^{7,14,19)}$. However, the presence of cerebral arterial stenosis did not differ between patients with lobar and non-lobar ICH in the present study, and further larger sample size study is required to elucidate the association between cerebral arterial stenosis and location of $\mathrm{ICH}$.

ICAS is more prevalent in Asian, and black people than in Caucasians. As well, small vessel disease such as hypertensive ICH and lacunar infarcts are more prevalent in Asians. In keeping with this epidemiologic association, patients with lacunar stroke often experience hemorrhagic stroke ${ }^{4,10)}$. The reasons for differing prevalence of ICAS, lacunar infarct, and $\mathrm{ICH}$ among populations remain unknown. Hypertension is the most important risk factor for small vessel disease and, it has been suggested that the role of hypertension may be greater in ICAS than in ECAS ${ }^{5,23)}$. Therefore, it can be speculated that hypertensive vascular damage might be the factor linking ICAS and ICH.

There are several limitations to our study. First, this was a retrospective cross-sectional analysis. Therefore, whether the presence of cerebral arterial stenosis implicate poor vascular prognosis or not could not be addressed and remains to be determined by further prospective investigation. Second, because the sample size was relatively small, the association of arterial stenosis and location of ICH was not determined in the present study. Further studies with a larger sample size are required to analyze the relation of $\mathrm{ICH}$ pattern and arterial stenosis. Also, because of the relative scarcity of patients with extracranial carotid stenosis, we did not analyze the characteristics of patients with ICH and extracranial carotid stenosis. Third, other causes of arterial stenosis such as vasospasm after ICH could not be excluded because follow-up angiography was not done in most patients. Finally, although we investigated the prevalence of ICAS and ECAS in patients with ICH, prevalence of ICAS and ECAS in non-stroke population was not available in the present study. Therefore, significance of presence of arterial stenosis in ICH population compared to non-stroke population could not be clearly determined in the present study. Further study enrolling subjects with $\mathrm{ICH}$, ischemic stroke, and non-stroke population are required to clarify the association between ICH and cerebral arterial stenosis.

\section{CONCLUSION}

This study showed that $19.2 \%$ of patients with spontaneous ICH had ICAS, but the prevalence of ECAS was relatively lower $(7.2 \%)$ compared with ICAS. Aging and diabetes are factors associated with the presence of ICAS, and aging, diabetes, and hypertension are associated with the presence of cerebral arterial stenosis in patients with $\mathrm{ICH}$. Because stroke recurrence could present as either ischemic or hemorrhagic stroke, further studies regarding the predictors of different subtypes of recurrent stroke should be undertaken in patients with $\mathrm{ICH}$.

\section{References}

1. Arenillas JF, Candell-Riera J, Romero-Farina G, Molina CA, Chacon P, Aguade-Bruix $S$, et al. : Silent myocardial ischemia in patients with symptomatic intracranial atherosclerosis: associated factors. Stroke 36 : 1201-1206, 2005

2. Bailey RD, Hart RG, Benavente O, Pearce LA : Recurrent brain hemorrhage is more frequent than ischemic stroke after intracranial hemorrhage. Neurology 56 : 773-777, 2001

3. Bang OY : Intracranial atherosclerosis: current understanding and perspectives. J Stroke $16: 27-35,2014$

4. Bang OY : Considerations when subtyping ischemic stroke in Asian patients. J Clin Neurol 12 : 129-136, 2016

5. Caplan LR, Gorelick PB, Hier DB : Race, sex and occlusive cerebrovascular disease: a review. Stroke $17:$ 648-655, 1986

6. Chimowitz MI, Lynn MJ, Derdeyn CP, Turan TN, Fiorella D, Lane BF, et 
Intracerebral Hemorrhage and Cerebral Arterial Stenosis | Chung PW, et al.

al. : Stenting versus aggressive medical therapy for intracranial arterial stenosis. N Engl J Med 365 : 993-1003, 2011

7. Hanger HC, Wilkinson TJ, Fayez-Iskander N, Sainsbury R : The risk of recurrent stroke after intracerebral haemorrhage. J Neurol Neurosurg Psychiatry 78 : 836-840, 2007

8. Hill MD, Silver FL, Austin PC, Tu JV : Rate of stroke recurrence in patients with primary intracerebral hemorrhage. Stroke 31 : 123-127, 2000

9. Inagawa $T$ : Recurrent primary intracerebral hemorrhage in Izumo City, Japan. Surg Neurol 64 : 28-35; discussion 35-36, 2005

10. Jackson C, Sudlow $C$ : Comparing risks of death and recurrent vascular events between lacunar and non-lacunar infarction. Brain 128(Pt 11) : 2507-2517, 2005

11. Koo $\mathrm{J}$ : The latest information on intracranial atherosclerosis: diagnosis and treatment. Interv Neurol $4: 48-50,2015$

12. Kremer PH, Jolink WM, Kappelle LJ, Algra A, Klijn CJ; SMART and ESPRIT Study Groups : Risk factors for lobar and non-lobar intracerebral hemorrhage in patients with vascular disease. PLoS One 10 : e0142338, 2015

13. Levey AS, Bosch JP, Lewis JB, Greene T, Rogers N, Roth D : A more accurate method to estimate glomerular filtration rate from serum creatinine: a new prediction equation. Modification of Diet in Renal Disease Study Group. Ann Intern Med 130 : 461-470, 1999

14. Li XQ, Su DF, Chen HS, Fang Q : Clinical neuropathological analysis of 10 cases of cerebral amyloid angiopathy-related cerebral lobar hemorrhage.

J Korean Neurosurg Soc 58 : 30-35, 2015

15. Logallo N, Naess H, Waje-Andreassen U, Thomassen L : Prevalence of intracranial stenosis in a Norwegian ischemic stroke population.

J Stroke Cerebrovasc Dis 23 : 1611-1615, 2014

16. Matsui R, Nakagawa T, Takayoshi H, Onoda K, Oguro H, Nagai A, et al. : A prospective study of asymptomatic intracranial atherosclerotic stenosis in neurologically normal volunteers in a Japanese cohort. Front Neurol $7: 39,2016$

17. Mazighi M, Tanasescu R, Ducrocq X, Vicaut E, Bracard S, Houdart E, et al. : Prospective study of symptomatic atherothrombotic intracranial stenoses: the GESICA study. Neurology 66 : 1187-1191, 2006

18. Nahab F, Cotsonis G, Lynn M, Feldmann E, Chaturvedi S, Hemphill JC, et al. : Prevalence and prognosis of coexistent asymptomatic intracranial stenosis. Stroke 39 : 1039-1041, 2008

19. Nakase T, Yoshioka S, Sasaki M, Suzuki A : Clinical features of recurrent stroke after intracerebral hemorrhage. Neurol Int 4 : e10, 2012

20. Qureshi Al, Mendelow AD, Hanley DF : Intracerebral haemorrhage. Lancet 373 : 1632-1644, 2009

21. Samuels OB, Joseph GJ, Lynn MJ, Smith HA, Chimowitz MI : A standardized method for measuring intracranial arterial stenosis. AJNR Am J Neuroradiol 21 : 643-646, 2000

22. Sato S, Uehara T, Hayakawa M, Nagatsuka K, Minematsu K, Toyoda K : Intra- and extracranial atherosclerotic disease in acute spontaneous intracerebral hemorrhage. J Neurol Sci 332 : 116-120, 2013

23. Uehara $T$, Tabuchi M, Mori E : Frequency and clinical correlates of occlusive lesions of cerebral arteries in Japanese patients without stroke. Evaluation by MR angiography. Cerebrovasc Dis 8 : 267-272, 1998

24. Wong KS, Ng PW, Tang A, Liu R, Yeung V, Tomlinson B : Prevalence of asymptomatic intracranial atherosclerosis in high-risk patients. Neurology 68 : 2035-2038, 2007

25. Wong LK : Global burden of intracranial atherosclerosis. Int J Stroke 1 : 158-159, 2006

26. Zia E, Engström G, Svensson PJ, Norrving B, Pessah-Rasmussen $H$ : Three-year survival and stroke recurrence rates in patients with primary intracerebral hemorrhage. Stroke 40 : 3567-3573, 2009 